\title{
Effects of Exercise Training on Neuromuscular Junction Morphology and Pre- to Post-Synaptic Coupling in Young and Aged Rats
}

\author{
M. R. Deschenes \\ Coll William \& Mary, Dept Kinesiol \& HIth Sci, Williamsburg, VA 23187 USA \\ R. N. Garratt \\ Coll William \& Mary, Dept Kinesiol \& HIth Sci, Williamsburg, VA 23187 USA \\ C. M. Leathrum \\ Coll William \& Mary, Dept Kinesiol \& HIth Sci, Williamsburg, VA 23187 USA \\ E. C. Shaffrey \\ Coll William \& Mary, Dept Kinesiol \& HIth Sci, Williamsburg, VA 23187 USA \\ K. A. Kressin \\ Coll William \& Mary, Program Neurosci, Williamsburg, VA 23187 USA
}

Follow this and additional works at: https://scholarworks.wm.edu/aspubs

\section{Recommended Citation}

Deschenes, M. R., Maresh, C. M., Crivello, J. F., Armstrong, L. E., Kraemer, W. J., \& Covault, J. (1993). The effects of exercise training of different intensities on neuromuscular junction morphology. Journal of neurocytology, 22(8), 603-615. 


\section{EFFECTS OF EXERCISE TRAINING ON NEUROMUSCULAR JUNCTION MORPHOLOGY AND PRE- TO POST-SYNAPTIC COUPLING IN YOUNG AND AGED RATS}

\author{
M. R. DESCHENES, ${ }^{a, b *}$ K. A. KRESSIN, ${ }^{b}$ \\ R. N. GARRATT, ${ }^{a}$ C. M. LEATHRUM ${ }^{a}$ AND \\ E. C. SHAFFREY \\ a Department of Kinesiology \& Health Sciences, College of William \\ \& Mary, Williamsburg, VA 23187-8795, USA \\ ${ }^{\mathrm{b}}$ Program in Neuroscience, College of William \& Mary, Williamsburg, \\ VA 23187-8795, USA
}

\begin{abstract}
The objective of this study was to determine whether pre- to post-synaptic coupling of the neuromuscular junction (NMJ) could be maintained in the face of significant morphological remodeling brought about by exercise training, and whether aging altered this capacity. Eighteen young adult $(8 \mathrm{mo})$ and eighteen aged $(24 \mathrm{mo})$ Fischer 344 rats were randomly assigned to either endurance trained (treadmill running) or untrained control conditions resulting in four groups $(N=9 /$ group). After the 10 -week intervention rats were euthanized and hindlimb muscles were surgically removed, quickly frozen at approximate resting length and stored at $-85^{\circ} \mathrm{C}$. The plantaris and EDL muscles were selected for study as they have different functions (ankle extensor and ankle flexor, respectively) but both are similarly and overwhelmingly comprised of fasttwitch myofibers. NMJs were stained with immunofluorescent procedures and images were collected with confocal microscopy. Each variable of interest was analyzed with a 2-way ANOVA with main effects of age and endurance training; in all cases significance was set at $P \leqslant 0.05$. Results showed that no main effects of aging were detected in NMJs of either the plantaris or the EDL. Similarly, endurance training failed to alter any synaptic parameters of EDL muscles. The same exercise stimulus in the plantaris however, resulted in significant pre- and post-synaptic remodeling, but without altering pre- to post-synaptic coupling of the NMJs. Myofiber profiles of the same plantaris and EDL muscles were also analyzed. Unlike NMJs, myofibers displayed significant age-related atrophy in both the plantaris and EDL muscles. Overall, these results confirm that despite significant training-induced reconfiguration of NMJs, pre- to post-synaptic coupling remains intact underscoring the importance of maintaining proper apposition of neurotransmitter release and binding sites so that effective
\end{abstract}

\footnotetext{
${ }^{*}$ Correspondence to: M.R. Deschenes, Department of Kinesiology \& Health Sciences, College of William \& Mary, Williamsburg, VA 23187 8795, USA. Tel: +1-757-221-2778; fax: +1-757-221-2761. E-mail address: mrdesc@wm.edu (M. R. Deschenes).

Abbreviations: ACh, acetylcholine; BSA, bovine serum albumin; BTX, $\alpha$-bungarotoxin; NMJ, neuromuscular junction; PBS, phosphatebuffered saline.
} nerve to muscle communication is assured.
(c) 2015 IBRO. Published by Elsevier Ltd. All rights reserved.

Key words: synapse, endplate, myofiber, aging, nerve terminal, NMJ.

\section{INTRODUCTION}

Previous research has demonstrated that the neuromuscular junction (NMJ) has the capacity to undergo considerable morphological remodeling as a result of increased activity presented in the form of exercise training. For example, endurance training has been shown to increase the total length of nerve terminal branching (Andonian and Fahim, 1988; Waerhaug et al., 1992), the area occupied by acetylcholine (ACh)-containing vesicles (Deschenes et al., 1993), the area occupied by ACh receptors (Cheng et al., 2013; Fahim, 1997; Gyorkos and Spitsbergen, 2014), and the dispersion of both pre-synaptic ACh vesicles and post-synaptic ACh receptors (Deschenes et al., 1993, 2000).

Similarly, it has been established that the natural process of aging also results in significant structural remodeling of the NMJ. Typically, aging is associated with adaptations such as increased pre-synaptic branching, post-synaptic endplate area, and increased separation of clusters of pre-synaptic vesicles and postsynaptic receptors, along with receptors that have been abandoned by previously linked nerve terminal branch points (see reviews by Gonzalez-Freire et al., 2014; Jang and Van Remmen, 2011; Rudolf et al., 2014).

More recently it has been reported that despite such extensive NMJ remodeling, the coupling of the pre- and post-synaptic components of the NMJ remains steadfast (Deschenes et al., 2013). In that investigation it was revealed that pre- to post-synaptic coupling remained constant despite the slow and progressive effects of the aging process, and the natural variation in activity levels characterizing different muscle groups (i.e., postural vs. non-weight bearing). To date, however, the impact of exercise training - which dramatically and abruptly increases neuromuscular activity - on pre- to postsynaptic coupling of the NMJ has yet to be determined. With that in mind, the present study was conducted to assess how endurance training affected NMJ morphology 
with a special emphasis on synaptic coupling of nerve terminal branches, ACh vesicles and post-synaptic ACh receptors.

\section{EXPERIMENTAL PROCEDURES}

\section{Subjects}

Eighteen young adult (8 mo old) and eighteen aged ( 24 mo old) male Fischer 344 rats were purchased from the National Institute of Aging Colonies. According to Turtorro et al. (1999) the average lifespan of male Fischer 344 rats is 25.5 mo while the average lifespan of men in the United States is 75.2 years (Arias, 2006). Accordingly, the rats used in the present study would roughly be the equivalent of 71-year-old men. Different muscles, i.e., soleus, from these same animals had been the subject of an earlier report from this laboratory (Deschenes et al., 2011).

Animals were provided standard rat chow and water ad libitum and were housed in a $21-22^{\circ} \mathrm{C}$ environment with a 12-h light/dark cycle throughout the duration of the investigation. All procedures were approved beforehand by the Institutional Animal Care and Use Committee, which adheres to the National Institutes of Health Guide for the Care and Use of Laboratory Animals as revised in 2011. Throughout the study, all efforts were made to minimize the number of animals used and to alleviate their discomfort.

\section{Exercise training}

Rats from both age groups were randomly assigned to either endurance training, or control groups resulting in a total of four treatment groups each with $N=9$. Endurance training consisted of a 10-week running program on a motorized treadmill (Accuscan Instruments, Columbus, OH, USA). The program began with training sessions of $15 \mathrm{~min}$ at a speed of $7.5 \mathrm{~m} / \mathrm{min}$ at a $0 \%$ grade completed 5 days/week. Treadmill speed and duration of exercise sessions were gradually increased such that by the final week, young and aged rats ran for 60-min per session at a speed of $15 \mathrm{~m} / \mathrm{min}$, while maintaining a $0 \%$ grade, and still for 5 days/week. Increments in exercise speed and duration were determined by the tolerance of aged animals, and replicated by young ones, to ensure that both age groups completed the same training regimen. Exercise tolerance was subjectively determined by visual indications of physical fatigue such as decisively labored strides, heavy panting, and inability to maintain pace. Aged and young adult controls remained sedentary and stayed in their tubs throughout the 10-week intervention period.

\section{Tissue preparation and storage}

At the conclusion of the 10-week intervention period, animals were euthanized and hindlimb muscles were dissected out, cleared of fat and connective tissue, quickly frozen at resting length in isopentane chilled with liquid nitrogen and stored at $-85^{\circ} \mathrm{C}$ until analysis. For the present study, the plantaris and EDL muscles were used for analysis. Although both of these muscles are principally comprised of fast-twitch (type II) muscle fibers (Delp and Duan, 1996), they have different functions in that the plantaris is an ankle extensor while the EDL is an ankle flexor.

\section{Cytofluorescent staining of NMJs}

To visualize NMJs, $50-\mu \mathrm{m}$-thick longitudinal sections of the middle one-third of the muscle were obtained at $-20^{\circ} \mathrm{C}$ on a cryostat (Cryocut 1800; Reichert-Jung, NuBloch, Germany). To prevent contraction of sections, microscope slides were pretreated in a $3 \%$ EDTA solution as previously described (Pearson and Sabarra, 1974). Sections were washed $4 \times 15 \mathrm{~min}$ in phosphatebuffered saline (PBS) containing $1 \%$ bovine serum albu$\min$ (BSA). Sections were then incubated in a humidified chamber overnight at $4{ }^{\circ} \mathrm{C}$ in supernatant of the primary antibody RT97 (Developmental Studies Hybridoma Bank, University of lowa), diluted 1:20 in PBS with 1\% BSA. The RT97 antibody reacts with non-myelinated segments of pre-synaptic nerve terminals (Anderton et al., 1982). The next day, sections were washed $4 \times 15 \mathrm{~min}$ in PBS with $1 \% \mathrm{BSA}$ before being incubated for $2 \mathrm{~h}$ at room temperature in fluorescein isothiocyanate (FITC)-conjugated secondary immunoglobulin (Sigma Chemical, St. Louis, MO, USA) which had been diluted 1:150 in PBS with $1 \%$ BSA. Sections were then washed $4 \times 15 \mathrm{~min}$ in PBS with $1 \%$ BSA before being incubated in a humidified chamber overnight at $4{ }^{\circ} \mathrm{C}$ in a solution containing rhodamine-conjugated $\alpha$-bungarotoxin (BTX; Molecular Probes, Eugene, OR, USA) diluted 1:600 in PBS, along with anti-synaptophysin (MP Biomedicals, Solon, $\mathrm{OH}$, USA) at a dilution of 1:50. BTX recognizes post-synaptic ACh receptors, while anti-synaptophysin binds to membranes of pre-synaptic vesicles containing ACh. Indeed, synaptophysin is the most abundant protein found in the synaptic vesicular membrane (Kwon and Chapman, 2011). The next day, sections were washed $4 \times 15 \mathrm{~min}$ in PBS with $1 \%$ BSA before incubating them for $2 \mathrm{~h}$ in a humidified chamber at room temperature in AlexaFluor 647 (Molecular Probes, Eugene, OR, USA)-labeled secondary antibody to illuminate the anti-synaptophysin. Sections were then washed again for $4 \times 15 \mathrm{~min}$ before being lightly coated with Pro Long (Molecular probes, Eugene, OR, USA) and having cover slips applied. Slides were then coded with respect to treatment group to allow for blinded evaluation of NMJ morphology and then stored at $-20^{\circ} \mathrm{C}$ until analysis. An example of this cytofluorescent staining of pre- and post-synaptic components of the NMJ is displayed in Fig. 1.

Pre-synaptic variables of NMJs assessed included: (1) number of branches identified at the nerve terminal, (2) the total length of those branches, (3) average length per branch, and (4) branching complexity which, as described by Tomas et al. (1990) is derived by multiplying the number of branches by the total length of those branches and dividing that figure by 100 . Pre-synaptic vesicular staining was assessed as: (1) total perimeter, or the length encompassing the entire vesicular region comprised of stained vesicular clusters and non-stained regions interspersed within those clusters, (2) stained 

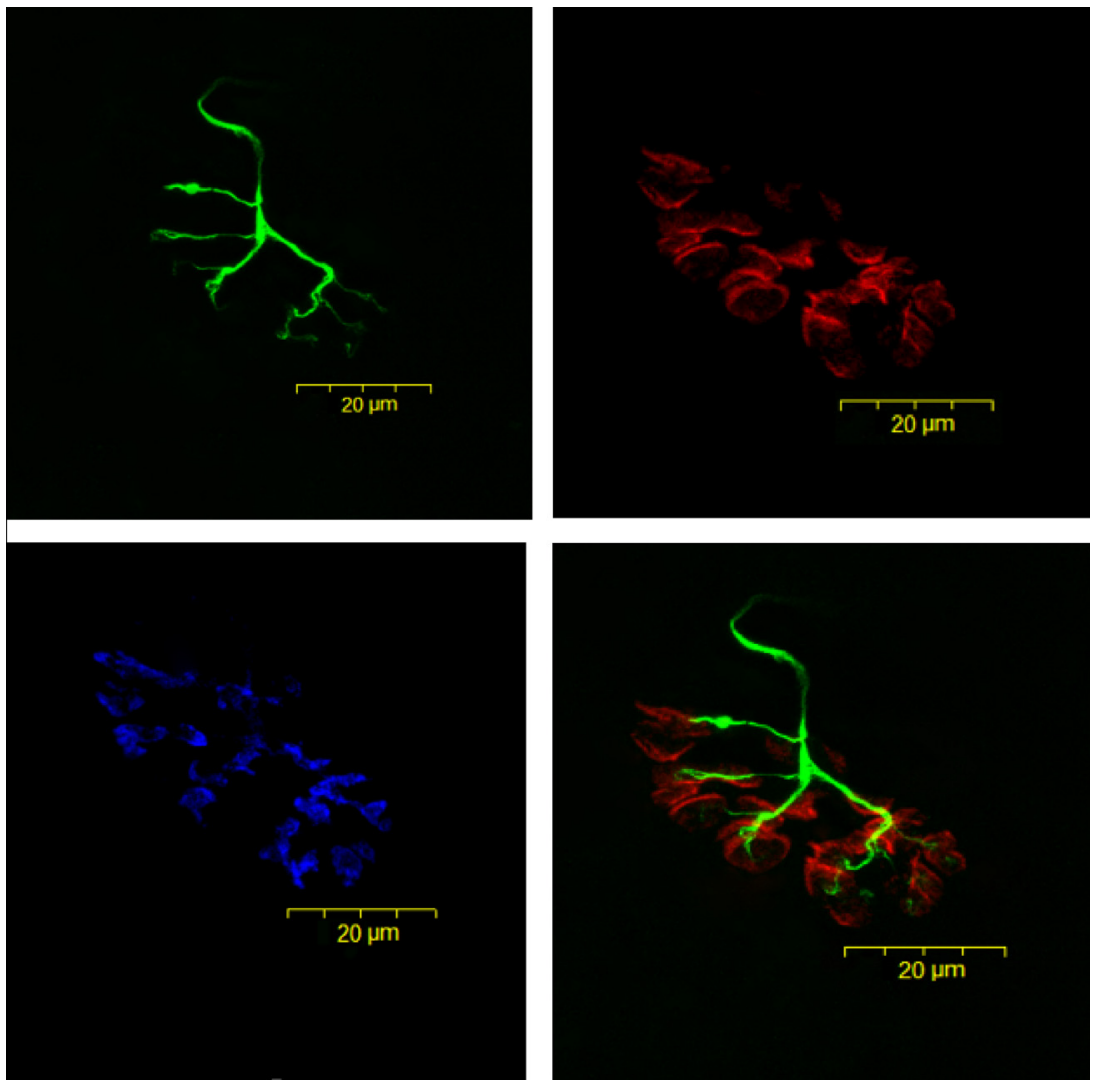

Fig. 1. Representative panel of images of the same fluorescently stained neuromuscular junction from young, control plantaris muscle. Presynaptic nerve terminal branches are stained in green, post-synaptic ACh receptors are stained in red, and pre-synaptic ACh vesicles are stained in blue. Original magnification of $\times 1000$ (scale bar $=20 \mu \mathrm{m}$ ). (For interpretation of the references to color in this figure legend, the reader is referred to the web version of this article.)

perimeter, or the composite length of tracings around individual clusters of vesicles, (3) total area, which includes stained vesicles along with non-stained regions interspersed among vesicle clusters, (4) stained area, or the cumulative areas occupied by $\mathrm{ACh}$ vesicular clusters, and (5) dispersion of vesicles, which was assessed by dividing the vesicular stained area by its total area and multiplying by 100 . Post-synaptic variables of interest included: (1) total perimeter, or the length encompassing the entire endplate comprised of stained receptor clusters and non-stained regions interspersed within those clusters, (2) stained perimeter, or the composite length of tracings around individual receptor clusters, (3) total area, which includes stained receptors along with non-stained regions interspersed among receptor clusters, (4) stained area, or the cumulative areas occupied by ACh receptor clusters, and (5) dispersion of endplates, which was assessed by dividing the endplate's stained area by its total area and multiplying by 100 . See Fig. 2 to observe pre- and post-synaptic tracings around $\mathrm{ACh}$ vesicles and receptors that were generated both manually and by software to assess total and stained perimeter lengths, respectively, and areas contained within those perimeter lengths. The same figure also shows manually traced lengths of branches of nerve terminal endings. In this study, pre- to post-synaptic coupling was quantified by dividing the NMJ's post-synaptic stained area by its total length of nerve terminal branching, as well as by quantifying the percentage of the area of post-synaptic staining of receptors that was overlapped with staining of presynaptic ACh vesicles. Finally, to approximate the number of ACh receptors supported by a given length of pre-synaptic nerve terminal branch length, the stained area of occupied by vesicles was divided by the total length of branching for that NMJ. An example of these tracings around features of the NMJ are presented in Fig. 2.

\section{Immunohistochemical staining of myofibers}

To quantify muscle fiber profiles (fiber size and fiber type), 10- $\mu \mathrm{m}$-thick transverse sections were obtained from the midbelly of the muscle using a cryostat (Cryocut 1800, Reichert-Jung, NuBloch, Germany) set at $-20^{\circ} \mathrm{C}$. In preparation for staining, four consecutive sections of the same muscle were collected and placed in series on a single microscope slide. This allowed each section to be stained for a single myofiber type. Microscope slides were coded with respect to treatment group to avoid measurement bias. In identifying different fiber types, each section was first incubated at room temperature for one hour in either the BA-D5, SC-71, 6H1, or F3 primary antibodies which uniquely interact with the type I, IIA, IIX, or IIB myosin heavy chains, respectively 

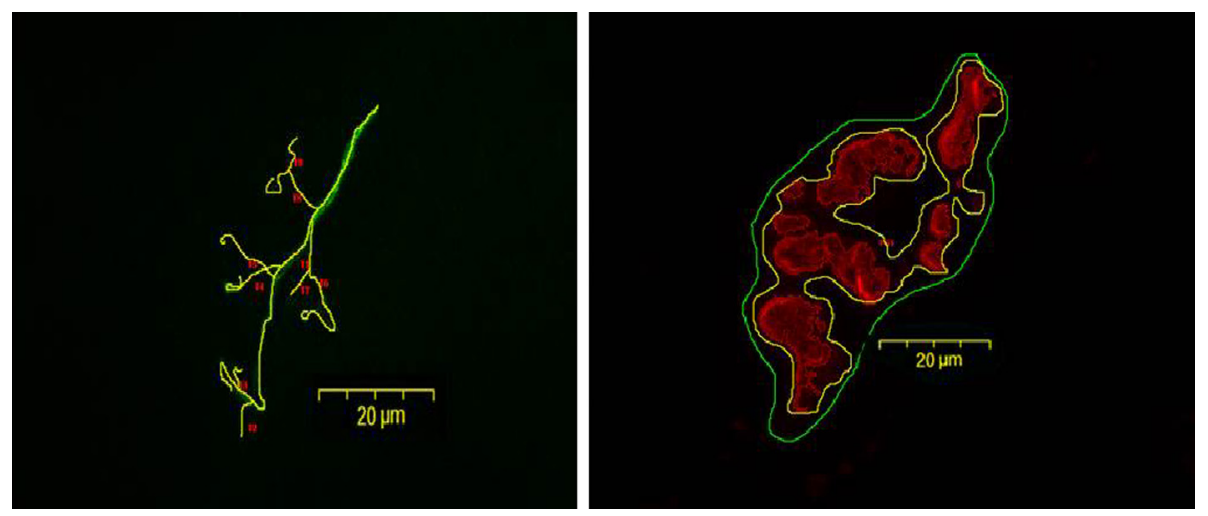

Fig. 2. Representative image of tracings used to quantify morphological aspects of neuromuscular junction from aged, control plantaris muscle. The left panel depicts tracings used to quantify presynaptic nerve terminal branches. The right panel illustrates tracings (both manually drawn and generated by software) used to quantify postsynaptic ACh receptors. Original magnification of $\times 1000$ (scale bar $=20 \mu \mathrm{m}$ ).

(National Institutes of Health Hybridoma Bank, lowa City, IA, USA). Subsequently, sections were washed with PBS and staining was carried out using a horse radish peroxidase, anti-mouse imaging system according to the manufacturer's instructions (ScyTek Laboratories, Logan, UT, USA). A representative image of the staining procedure is presented in Fig. 3.

\section{Microscopy}

An Olympus Fluoview FV 300 confocal system featuring three lasers and an Olympus BX60 fluorescent microscope (Olympus America, Melville, NY, USA) was used to collect images of NMJs. Using a $100 \times$ oil immersion objective, it was initially established that the entire NMJ was within the longitudinal borders of the myofiber and that damage to the structure had not occurred during sectioning. A detailed image of the entire NMJ was constructed from a z-series of scans taken at $1-\mu \mathrm{m}$-thick increments. Digitized, twodimensional images of NMJs were stored on the system's hard drive and later quantified with the ImagePro Plus software (Media Cybernetics, Silver Spring, MD, USA). A minimum of 10 NMJs per muscle were quantified and measurements were averaged to represent synaptic morphology within that muscle.

In the quantification of muscle fiber profiles, an Olympus BX41 bright field microscope was used in conjunction with a $40 \times$ objective. Myofiber crosssectional areas were quantified with the Image ProExpress software. A random sample of 150-200 myofibers from each muscle was analyzed to determine average myofiber size (i.e., cross-sectional area) and fiber-type composition ( $\%$ of total number of fibers examined) for that muscle.
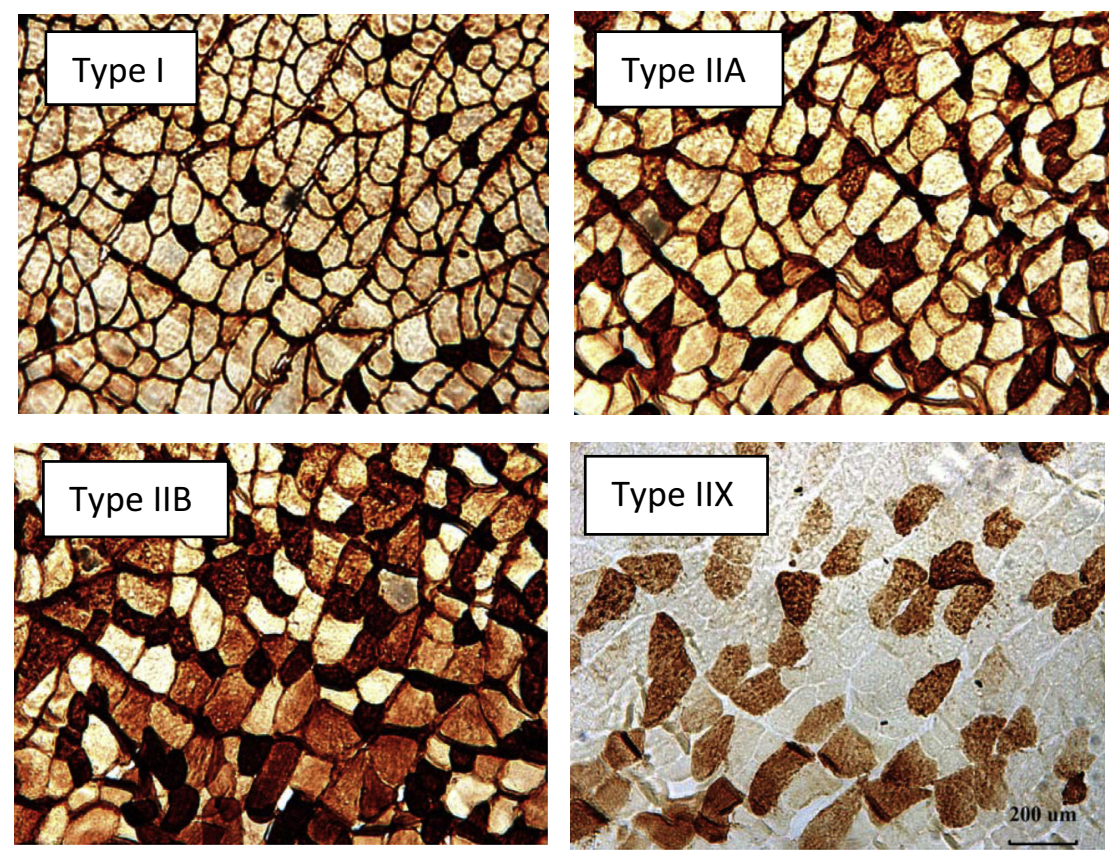

Fig. 3. Plantaris myofibers stained with immunohistochemical procedures. Each panel shows staining of a particular fiber type. Original magnification of $\times 100$. 


\section{Statistical analysis}

Data are reported as mean \pm SE. A two-way ANOVA with main effects of age (young adult vs. aged) and treatment (endurance trained vs. control) was performed on each NMJ and myofiber variable of interest. In the event of a significant $F$ ratio for either a main effect or an interactive one, a Fisher-Protected Least Squared Difference (PLSD) post-hoc analysis procedure was conducted to identify significant pair-wise differences. Significance was set at $P \leqslant 0.05$.

\section{RESULTS}

\section{Body mass}

Prior to the 10-week intervention period our ANOVA results revealed a significant main effect of age (Aged > Young). Indeed, post-hoc analysis indicated that regardless of treatment group, older rats weighed significantly more than young adult ones. No main effect for treatment group, however, was identified at the preintervention interval. In contrast, our follow-up analysis conducted at the end of the intervention period showed that a main effect for treatment had been established as a result of the 10-week training program. In both aged and young adult rats, treadmill running was associated with a lower body mass than that noted among untrained control rats. These results are presented in Table 1.

\section{NMJ morphology}

Plantaris. Our morphological data collected on NMJs in this fast-twitch ankle extensor revealed significant training-induced pre- and post-synaptic NMJ remodeling. Pre-synaptically, there was a main effect of training whereby treadmill running resulted in a greater number of nerve terminal branches per NMJ. However, post-hoc analysis demonstrated that this traininginduced response relative to age-matched controls was apparent only among young adult NMJs. Similarly, it was established that although a main effect of training was detected with respect to total nerve terminal branch length per NMJ, post-hoc results showed that only in younger animals did endurance training promote an enhanced total branch length. The combination of branch number and total length of branching led to similar results regarding branching complexity. That is, relative to untrained controls, trained rats displayed greater branching complexity but only among young adult, and not older NMJs. In contrast to what was detected for number of nerve terminal branches and total branch length of the pre-synaptic region, the average length of individual nerve terminal branches was not altered by endurance training. And in none of the quantified variables of nerve terminal branching was there evidence of an effect of aging.

To more thoroughly assess the consequences of aging and endurance training on pre-synaptic NMJ morphology, synaptophysin contained in the membranes of ACh-containing vesicles was fluorescently stained and quantified. These findings revealed that the total perimeter of a line manually drawn around the clusters of ACh vesicles, as represented by synaptophysinspecific immunofluorescence, once again displayed a main effect of treadmill running (Trained $>$ Controls), and as before, post-hoc results showed that only among young adults did training elicit an expansion relative to untrained animals. When the area within those perimeter lines were analyzed, it too was established that total area - encompassing both clusters of vesicles and empty spaces interspersed among those clusters exhibited a main effect for training, but once again, this trained vs. control group expansion was noted only among young adult rats. This differed from our stained perimeter length and stained area measurements - in which aggregated lengths and areas of membranestained vesicles but not unstained regions between those vesicle clusters - where no significant effects of age or training were identified. The final two presynaptic parameters of interest, the dispersion of antisynaptophysin labeled $\mathrm{ACh}$ vesicles and the ratio of vesicular membrane-stained area to nerve terminal branch length, demonstrate significant main effects for endurance training, but not aging. More specifically, training was associated with a more dispersed distribution of vesicles but this adaptation was found to be significant only among young adults. Similarly, a significant main effect of training was found for the vesicular staining to branch length ratio suggesting that training led to a smaller number of vesicles anchored per given length of branching. Post-hoc analysis revealed that this adaptation once again existed in young adult, but not aged NMJs.

Assessment of post-synaptic ACh receptors also revealed a number of main effects of endurance training that post-hoc tests showed were manifested exclusively in young adult animals. For example, the total perimeter length of a single line manually drawn to encircle the entire endplate region (i.e. ACh receptor clusters and unstained regions between those clusters) was found to

Table 1. Body mass $(\mathrm{g})$ of young adult and aged rats before and after 10 weeks of endurance training

\begin{tabular}{llll}
\hline & Young, Control & Young, Trained & Aged, Control \\
\hline Before intervention & $379.6 \pm 7.1^{*}$ & $363.1 \pm 6.7^{*}$ & $398.8 \pm 6.5$ \\
After intervention & $456.8 \pm 10.9$ & $379.3 \pm 8.5^{\dagger}$ & $416.4 \pm 4.9$ \\
\hline
\end{tabular}

\footnotetext{
Values are mean $\pm \mathrm{SE}, N=9 /$ group.

* Indicates significant $(P \leqslant 0.05)$ difference from Aged, Control and Aged, Trained.

‡ Indicates significant $(P \leqslant 0.05)$ difference from Young, Control and Aged, Control.

$\dagger$ Indicates significant $(P \leqslant 0.05)$ difference from Young, Control.
} 
be expanded by treadmill running, but only among younger NMJs. This was also true of the cumulative perimeter lengths automatically generated by the imaging software solely around stained clusters of receptors, as it also was regarding the measurement of the total area of the endplate region inclusive not only of stained receptors, but also unstained regions between those receptor clusters. Conversely, when analyzing stained areas exclusively encompassing ACh receptors - and not unstained interspersing regions - no effects of either training or aging were identified. Yet in examining the distribution of those ACh receptors it was discovered that among young, but not aged NMJs endurance training resulted in a greater dispersion, or less compact, deposition of those receptors within the entirety of the post-synaptic endplate region.

Finally, our morphometric assessment indicated that pre- to post-synaptic coupling was unaffected by both endurance training and aging. This was found to be true whether pre- to post-synaptic coupling was viewed as the ratio of the stained receptor membrane area to nerve terminal branch length, or the degree of overlap among immunofluorescently labeled pre-synaptic vesicle membrane and post-synaptic receptors. All data related to the structure of NMJs within the plantaris muscle are presented in Table 2.

$E D L$. Analysis of the NMJs of the EDL muscle provided results similar to those of the plantaris muscle in that no main effect of aging was revealed in any of the morphological parameters of interest. But unlike the plantaris, NMJs of the EDL muscle were also impervious to the effects of endurance training as well as aging. Quantitative analysis of pre- and post-synaptic variables of the EDL can be found in Table 3.

\section{Myofiber morphology}

Plantaris. In assessing the effects of endurance training and aging on myofiber size within the plantaris, statistical results confirmed that with fiber types collapsed together, a significant main effect for training, but not aging was established. More specifically, without consideration of fiber type, the mean cross-sectional area of myofibers from aged control animals was significantly smaller than young control and young trained muscles. When investigating the size of Type I fibers alone, results indicated that those from young controls were significantly larger than Type I fibers from all other groups. And although no between-group differences in the size of Type IIA and IIX fibers were detected, it was noted that the Type IIB fibers of aged control plantaris muscles were significantly smaller than that same fiber type in each of the three other groups.

With respect to myofiber-type composition, it was determined that the percentage of Type I fibers expressed in aged trained plantaris muscles was significantly greater than in all three other experimental groups, while the percentage of Type IIA fibers was lesser in aged control muscles than in both the young

Table 2. Effects of aging and endurance training on neuromuscular junction morphology of plantaris muscles

\begin{tabular}{|c|c|c|c|c|}
\hline & Young, Control & Young, Trained & Aged, Control & Aged, Trained \\
\hline \multicolumn{5}{|l|}{ Pre-synaptic } \\
\hline Branch number & $9.2 \pm 1.1$ & $15.5 \pm 1.0^{*}$ & $11.6 \pm 1.1$ & $14.3 \pm 1.0$ \\
\hline Total branch length $(\mu \mathrm{m})$ & $105.9 \pm 14.3$ & $207.0 \pm 19.2^{*}$ & $148.3 \pm 15.8$ & $194.2 \pm 33.9$ \\
\hline Average branch length $(\mu \mathrm{m})$ & $21.1 \pm 8.4$ & $13.9 \pm 1.0$ & $14.7 \pm 2.0$ & $13.1 \pm 1.1$ \\
\hline Branching complexity & $11.8 \pm 2.9$ & $39.5 \pm 6.1^{*}$ & $20.9 \pm 4.8$ & $35.8 \pm 9.8$ \\
\hline Vesicles stained area/total branch length & $1.1 \pm 0.2$ & $0.7 \pm 0.1^{*}$ & $1.0 \pm 0.1$ & $0.7 \pm 0.1^{*}$ \\
\hline Total vesicles perimeter $(\mu \mathrm{m})$ & $112.6 \pm 6.1$ & $166.4 \pm 11.0^{*}$ & $136.9 \pm 7.5$ & $145.3 \pm 145.3$ \\
\hline Stained vesicles perimeter $(\mu \mathrm{m})$ & $184.3 \pm 15.8$ & $233.9 \pm 32.6$ & $208.4 \pm 18.2$ & $202.8 \pm 24.8$ \\
\hline Total area vesicles $\left(\mu \mathrm{m}^{2}\right)$ & $463.1 \pm 39.4$ & $829.4 \pm 100.2^{*}$ & $604.4 \pm 55.2$ & $692.1 \pm 67.2$ \\
\hline Stained area vesicles $\left(\mu \mathrm{m}^{2}\right)$ & $92.2 \pm 15.8$ & $126.2 \pm 26.7$ & $111.5 \pm 14.0$ & $108.2 \pm 18.4$ \\
\hline Vesicle dispersion (\%) & $25.2 \pm 5.8^{\dagger}$ & $15.8 \pm 1.8$ & $20.2 \pm 2.0$ & $16.0 \pm 1.6$ \\
\hline \multicolumn{5}{|l|}{ Post-synaptic } \\
\hline Total receptors perimeter $(\mu \mathrm{m})$ & $117.7 \pm 4.2^{\#}$ & $169.6 \pm 10.2^{\S}$ & $140.6 \pm 8.1$ & $145.5 \pm 7.4$ \\
\hline Stained receptors perimeter $(\mu \mathrm{m})$ & $211.1 \pm 14.2$ & $340.6 \pm 32.7^{\S}$ & $236.9 \pm 15.2$ & $250.5 \pm 16.7$ \\
\hline Total area receptors $\left(\mu \mathrm{m}^{2}\right)$ & $538.3 \pm 66.7$ & $1019.2 \pm 93.7^{\S}$ & $688.7 \pm 56.1$ & $701.5 \pm 43.7$ \\
\hline Stained area receptors $\left(\mu \mathrm{m}^{2}\right)$ & $205.4 \pm 21.0$ & $252.1 \pm 29.5$ & $208.6 \pm 16.2$ & $209.1 \pm 20.2$ \\
\hline Receptor dispersion (\%) & $41.4 \pm 2.2$ & $27.4 \pm 2.0$ & $34.7 \pm 2.5$ & $34.1 \pm 4.6$ \\
\hline Receptors stained area/total branch length & $2.3 \pm 0.3$ & $1.5 \pm 0.1$ & $1.8 \pm 0.1$ & $2.0 \pm 0.6$ \\
\hline Receptor/vesicle coupling (\%) & $64.4 \pm 15.9$ & $64.7 \pm 19.2$ & $62.2 \pm 9.2$ & $70.9 \pm 15.8$ \\
\hline
\end{tabular}

Values are mean $\pm \mathrm{SE}, N=9 /$ group.

Branching complexity $=$ branch number $\times$ total branch length $/ 100$

Vesicle dispersion $=$ stained area/total area $\times 100$ (lower $\%$ indicates greater dispersion)

Receptor dispersion $=$ stained area/total area $\times 100$ (lower \% indicates greater dispersion). Receptor/vesicle coupling $=$ vesicle stained area/receptor stained area $\times 100$

(higher \% indicates greater degree of overlap between presynaptic vesicles and post-synaptic receptors).

Indicates significant $(P \leqslant 0.05)$ difference from Young, Control and Aged, Control.

Indicates significant $(P \leqslant 0.05)$ difference from Young, Control.

$\dagger$ Indicates significant $(P \leqslant 0.05)$ difference from Young, Trained and Aged, Trained.

$\S$ Indicates significant $(P \leqslant 0.05)$ difference from all other groups.

\# Indicates significant $(P \leqslant 0.05)$ difference from Aged, Control and Aged, Trained. 
Table 3. Effects of aging and endurance training on neuromuscular junction morphology of edl muscles

\begin{tabular}{|c|c|c|c|c|}
\hline & Young, Control & Young, Trained & Aged, Control & Aged, Trained \\
\hline \multicolumn{5}{|l|}{ Pre-synaptic } \\
\hline Branch number & $9.5 \pm 1.0$ & $11.3 \pm 0.9$ & $10.5 \pm 0.8$ & $9.1 \pm 0.8$ \\
\hline Total branch length $(\mu \mathrm{m})$ & $106.1 \pm 11.6$ & $112.9 \pm 12.3$ & $103.9 \pm 8.1$ & $97.5 \pm 5.6$ \\
\hline Average branch length $(\mu \mathrm{m})$ & $12.3 \pm 0.8$ & $11.0 \pm 0.7$ & $11.3 \pm 0.9$ & $12.3 \pm 0.9$ \\
\hline Branching complexity & $12.9 \pm 2.5$ & $15.8 \pm 2.9$ & $14.3 \pm 1.9$ & $10.8 \pm 1.6$ \\
\hline Vesicles stained area/total branch length & $1.0 \pm 0.1$ & $0.9 \pm 0.1$ & $1.1 \pm 0.1$ & $1.0 \pm 0.1$ \\
\hline Total vesicles perimeter $(\mu \mathrm{m})$ & $126.2 \pm 10.8$ & $129.1 \pm 10.8$ & $129.2 \pm 10.2$ & $123.2 \pm 3.6$ \\
\hline Stained vesicles perimeter $(\mu \mathrm{m})$ & $208.5 \pm 18.4$ & $202.7 \pm 25.9$ & $207.6 \pm 12.5$ & $209.7 \pm 18.2$ \\
\hline Total area vesicles $\left(\mu \mathrm{m}^{2}\right)$ & $525.7 \pm 72.6$ & $520.2 \pm 48.7$ & $569.7 \pm 39.7$ & $537.5 \pm 48.7$ \\
\hline Stained area vesicles $\left(\mu \mathrm{m}^{2}\right)$ & $90.2 \pm 15.4$ & $89.2 \pm 11.1$ & $96.6 \pm 9.1$ & $92.7 \pm 11.3$ \\
\hline Vesicle dispersion (\%) & $21.0 \pm 3.1$ & $21.7 \pm 3.5$ & $20.5 \pm 1.7$ & $19.5 \pm 1.6$ \\
\hline \multicolumn{5}{|l|}{ Post-synaptic } \\
\hline Total receptors perimeter $(\mu \mathrm{m})$ & $134.7 \pm 9.5$ & $133.2 \pm 11.3$ & $131.1 \pm 8.7$ & $125.0 \pm 5.3$ \\
\hline Stained receptors perimeter $(\mu \mathrm{m})$ & $257.2 \pm 21.1$ & $240.6 \pm 25.9$ & $252.6 \pm 18.0$ & $223.9 \pm 16.7$ \\
\hline Total area receptors $\left(\mu \mathrm{m}^{2}\right)$ & $659.6 \pm 74.5$ & $631.3 \pm 86.7$ & $637.5 \pm 48.3$ & $625.6 \pm 52.0$ \\
\hline Stained area receptors $\left(\mu \mathrm{m}^{2}\right)$ & $215.6 \pm 23.1$ & $220.7 \pm 24.3$ & $226.3 \pm 20.3$ & $212.4 \pm 30.5$ \\
\hline Receptor dispersion (\%) & $35.9 \pm 2.6$ & $38.8 \pm 1.8$ & $39.6 \pm 2.4$ & $38.3 \pm 2.9$ \\
\hline Receptors stained area/total branch length & $2.5 \pm 0.4$ & $2.2 \pm 0.2$ & $2.5 \pm 0.2$ & $2.4 \pm 0.3$ \\
\hline Receptor/vesicle coupling (\%) & $50.7 \pm 11.0$ & $45.2 \pm 6.1$ & $48.1 \pm 3.6$ & $51.4 \pm 3.4$ \\
\hline
\end{tabular}

Values are mean $\pm \mathrm{SE}, N=9 /$ group.

Branching complexity $=$ branch number $\times$ total branch length $/ 100$.

Vesicle dispersion $=$ stained area/total area $\times 100$ (lower \% indicates greater dispersion)

Receptor dispersion $=$ stained area/total area $\times 100$ (lower $\%$ indicates greater dispersion). Receptor/vesicle coupling $=$ vesicle stained area/receptor stained area $\times 100$ (higher \% indicates greater degree of overlap between pre-synaptic vesicles and post-synaptic receptors).

and aged trained groups. Type IIX fibers accounted for a greater percentage of total myofiber count in aged control plantaris muscles relative to all other groups while the percentage of Type IIB fibers in aged control muscles was discovered to be significantly lower than in young control muscles. These findings suggest that aging was linked with a conversion of Type IIB fibers to Type IIX.

The combination of data from fiber size and fiber-type composition was then used to calculate the relative area of whole muscle occupied by individual myofiber types in order to gain insights into expected functional features of the whole muscle. These findings revealed that in young trained rats Type I myofibers account for a smaller area of the whole muscle than they do in aged trained rats, and Type IIA fibers occupy a smaller relative area among aged control muscles than they do in aged trained ones. In those same aged control plantaris muscles it was documented that the area accounted for by Type IIX myofibers was greater than it was in the three other groups. Finally, it was confirmed that irrespective of treatment (i.e. control or trained), the area occupied by Type IIB fibers is significantly less in aged muscles than in young adult ones. All data concerning myofiber profiles of plantaris muscles are found in Table 4.

$E D L$. When examining the size of myofibers without regard to specific fiber type, it was determined that EDL myofibers of young control rats were significantly larger than those of the three other experimental groups. When analyzing the size of specific fiber types, it was noted that Type IIX myofibers of young controls were significantly larger than those found in both aged control and aged trained muscles, while the mean cross-sectional area of young control Type IIB fibers was greater than those fibers in aged trained muscles.

No differences in fiber-type composition were identified among the four experimental groups of EDL muscles, but the percentage of total area occupied by Type IIA fibers was less in aged control compared to aged trained muscles while in those same muscles (i.e. aged trained) Type IIB fibers occupied a smaller relative area than they did in young control rats. Data regarding EDL myofiber profiles are presented in Table 5.

\section{DISCUSSION}

The NMJ is capable of undergoing significant physiological and morphological remodeling throughout the lifespan. Indeed, the process of aging is accompanied by reconfiguration of the NMJ indicating a progressive abandonment of myofibers by their innervating motor neurons that is linked with an incomplete re-innervation of those same fibers by alternate, neighboring motor neurons (Robbins, 1992; Rosenheimer, 1990; Valdez et al., 2010). It has also been established that muscles and their constituent myofibers exhibit disparate NMJ structural features depending on natural recruitment patterns of muscles as well as their different myofiber-type profiles (Deschenes et al., 2015; Prakash et al., 1996). But despite such plasticity, it appears that proper coupling of the pre- and postsynaptic components of the NMJ remains intact to allow effective nerve to muscle communication, at least during the slow, gradual process of aging and differences in recruitment patterns that are endemic to normal daily postural and locomotor activities. In the present investigation we sought to expand our understanding of NMJ adaptability, with a particular focus on pre- to post-synaptic 
Table 4. Myofiber profiles of plantaris muscles

\begin{tabular}{|c|c|c|c|c|}
\hline & Young, Control & Young, Trained & Aged, Control & Aged, Trained \\
\hline \multicolumn{5}{|c|}{ Cross-sectional area $\left(\mu m^{2}\right)$} \\
\hline Types combined & $2033 \pm 173$ & $2025 \pm 205$ & $1508 \pm 76.9^{*}$ & $1655 \pm 193$ \\
\hline Type I & $1991 \pm 355^{\star}$ & $1069 \pm 134$ & $1141 \pm 78$ & $1223 \pm 68$ \\
\hline Type IIA & $1489 \pm 157$ & $1391 \pm 98$ & $1181 \pm 44$ & $1382 \pm 141$ \\
\hline Type IIX & $2048 \pm 105$ & $2303 \pm 285$ & $1700 \pm 121$ & $2138 \pm 327$ \\
\hline Type IIB & $2790 \pm 423$ & $3512 \pm 373$ & $1720 \pm 208$ & $2242 \pm 348^{\dagger}$ \\
\hline \multicolumn{5}{|c|}{ Fiber-type composition (\%) } \\
\hline Type I & $19.2 \pm 1.4$ & $16.7 \pm 2.8$ & $17.6 \pm 1.3$ & $26.0 \pm 3.1^{+}$ \\
\hline Type IIA & $27.5 \pm 1.6$ & $31.7 \pm 1.7$ & $23.4 \pm 1.8^{\S}$ & $30.0 \pm 2.1$ \\
\hline Type IIX & $34.8 \pm 2.6$ & $34.5 \pm 2.8$ & $47.6 \pm 2.7^{*}$ & $31.7 \pm 3.0$ \\
\hline Type IIB & $18.3 \pm 2.4$ & $16.9 \pm 2.8$ & $11.2 \pm 2.1^{\#}$ & $12.2 \pm 2.9$ \\
\hline \multicolumn{5}{|l|}{$\%$ area occupied } \\
\hline Type I & $18.5 \pm 2.9$ & $11.6 \pm 2.3^{\top}$ & $13.7 \pm 1.7$ & $20.7 \pm 3.0$ \\
\hline Type IIA & $20.5 \pm 1.8$ & $22.8 \pm 2.4$ & $18.8 \pm 1.9^{\circ}$ & $25.2 \pm 1.4$ \\
\hline Type IIX & $36.0 \pm 3.2$ & $38.1 \pm 2.9$ & $53.4 \pm 3.5^{*}$ & $39.3 \pm 3.9$ \\
\hline Type IIB & $24.8 \pm 3.4$ & $27.3 \pm 3.1$ & $13.8 \pm 3.0^{*}$ & $14.6 \pm 2.5^{*}$ \\
\hline
\end{tabular}

Values are mean $\pm \mathrm{SE}, N=9 /$ group.

* Indicates significant $(P \leqslant 0.05)$ difference from Young, Control and Young, Trained.

‡ Indicates significant $(P \leqslant 0.05)$ difference from all other groups.

† Indicates significant $(P \leqslant 0.05)$ difference from Young, Trained.

$\S$ Indicates significant $(P \leqslant 0.05)$ difference from Young, Trained and Aged, Trained.

\# Indicates significant $(P \leqslant 0.05)$ difference from Young, Control.

- Indicates significant $(P \leqslant 0.05)$ difference from Aged, Trained.

Table 5. Myofiber profiles of EDL muscles

\begin{tabular}{|c|c|c|c|c|}
\hline & Young, Control & Young, Trained & Aged, Control & Aged, Trainec \\
\hline \multicolumn{5}{|c|}{ Cross-sectional area $\left(\mu m^{2}\right)$} \\
\hline Types combined & $1827 \pm 171^{*}$ & $1505 \pm 197$ & $1459 \pm 127$ & $1169 \pm 123$ \\
\hline Type I & $937 \pm 101$ & $924 \pm 74$ & $759 \pm 54$ & $933 \pm 93$ \\
\hline Type IIA & $1155 \pm 75$ & $966 \pm 85$ & $936 \pm 58$ & $936 \pm 91$ \\
\hline Type IIX & $1730 \pm 151$ & $1444 \pm 138$ & $1274 \pm 124$ & $1150 \pm 96$ \\
\hline Type IIB & $2838 \pm 289^{*}$ & $2260 \pm 375$ & $2452 \pm 290$ & $1712 \pm 227$ \\
\hline \multicolumn{5}{|c|}{ Fiber-type composition (\%) } \\
\hline Type I & $9.8 \pm 1.7$ & $10.5 \pm 2.6$ & $9.6 \pm 1.3$ & $11.7 \pm 1.8$ \\
\hline Type IIA & $33.3 \pm 2.1$ & $32.8 \pm 2.9$ & $26.9 \pm 2.9$ & $34.0 \pm 3.3$ \\
\hline Type IIX & $26.3 \pm 3.8$ & $28.1 \pm 3.2$ & $35.0 \pm 3.5$ & $29.8 \pm 2.7$ \\
\hline Type IIB & $30.4 \pm 3.7$ & $28.5 \pm 4.1$ & $28.2 \pm 3.4$ & $24.3 \pm 4.6$ \\
\hline \multicolumn{5}{|l|}{$\%$ area occupied } \\
\hline Type I & $5.7 \pm 1.4$ & $8.7 \pm 3.1$ & $5.1 \pm 0.7$ & $10.0 \pm 2.1$ \\
\hline Type IIA & $21.5 \pm 1.5$ & $22.9 \pm 2.9$ & $18.6 \pm 2.9^{*}$ & $27.7 \pm 3.1$ \\
\hline Type IIX & $26.0 \pm 4.5$ & $27.8 \pm 3.2$ & $30.5 \pm 3.6$ & $29.8 \pm 2.9$ \\
\hline Type IIB & $46.6 \pm 5.7^{*}$ & $40.4 \pm 5.6$ & $45.5 \pm 4.0$ & $32.3 \pm 4.7$ \\
\hline
\end{tabular}

Values are mean $\pm \mathrm{SE}, N=9 /$ group.

* Indicates significant $(P \leqslant 0.05)$ difference from Aged, Trained.

$\star$ Indicates significant $(P \leqslant 0.05)$ difference from Aged, Control and Aged, Trained.

coupling synchronicity, when exposed to a far more vigorous, and abrupt, change in physical activity such as that experienced with exercise training, and to determine whether aging influenced such adaptability. In short, it was revealed that exercise training does indeed elicit morphological remodeling of the NMJ, but that this was dependent both on the recruitment pattern of the muscle, as well as the age of the animal. More specifically, it was demonstrated that endurance training promoted structural adaptations of the NMJ including enhanced nerve terminal branching length, number and complexity along with greater dispersion in the distribution of pre-synaptic ACh-containing vesicles to go along with post-synaptic modifications such as an expanded endplate area, and a more dispersed arrangement of ACh binding receptors. It is important to note, however, that these traininginduced NMJ modifications were found in the plantaris muscle, but not the EDL. Most likely this muscle specificity was due to the fact that although both these muscles are primarily composed of Type II, or fast-twitch myofibers, the plantaris is recruited roughly twice as much as the EDL during treadmill running in the rat (Glenn and Laughlin, 1987; Laughlin et al., 1988).

Our data also demonstrate, however, that these exercise-induced adaptations of the NMJ are evident in young adult, but not in aged animals. This was found to 
be true despite the fact that both young and aged rats completed the very same exercise training regimen, thus providing the same training stimulus. Previously, it has also been reported that exercise resulted in NMJ remodeling in younger rats but not in older ones, but that was accounted for by the natural remodeling that accompanies aging, thus leaving little potential for remodeling evoked by exercise training among younger animals (Deschenes et al., 2011). In the present study, however, aging by itself was not responsible for synaptic reconfiguration. In fact, not a single NMJ parameter quantified showed a significant main effect for aging and this was confirmed in both the plantaris and the EDL. Again, this contrasts with what was found in an earlier report examining the effects of training and aging on NMJs of the soleus where the impact of aging by itself led to the expansion of NMJ dimensions (Deschenes et al., 2011). Although the cause of this muscle specificity regarding the effects of aging cannot be known with certainty, it is possible that once again the effect of normal daily activity patterns of recruitment is involved as the postural soleus has been found to be recruited to a much higher extent than either the plantaris or the EDL during normal daily postural and ambulatory activity (Glenn and Laughlin, 1987; Laughlin et al., 1988).

It was of interest that in both young adult and aged NMJs, endurance training resulted in a greater number of nerve terminal branches which, in turn, led to greater total nerve terminal branch length while average branch length was unaffected. This phenomenon may best be explained by the observation that myelination of motor axons does not extend into the nerve terminal branches located at the NMJ that serve to anchor ACh-containing vesicles. Functionally, once myelination is no longer present at the nerve terminal endings, the action potentials radiating down the axons to the NMJ that ultimately trigger the exocytotic release of neurotransmitter, must - in the absence of myelination passively diffuse down the branch length. In doing so they progressively lose strength such that by the time they reach voltage-gated calcium channels, they may no longer be of sufficient intensity to open those channels, thus preventing the release of the neurotransmitter (Seidi, 2014). Consequently, it stands to reason that repeated firing and conduction of action potentials during exercise training would result in a larger number of terminal branches that remain at normal length in order to facilitate effective diffusion of electrical impulses into the NMJ. It should be noted that at this time, this explanation is speculative and awaits experimental confirmation.

Another interesting training-induced adaptation detected in young adult, as well as aged rats was the novel finding that per unit length of terminal branching, there was a decreased area of anti-synaptophysin staining, i.e., approximate number, of ACh vesicles. This aspect of synaptic remodeling is probably related to recent insights into the vesicular recycling that occur during neuromuscular activity (Kononenko and Haucke, 2015; Rizzoli, 2014). An integral part of this recycling is the re-uptake of the vesicular membrane that fuses with the terminal ending's membrane during exocytosis and release of ACh (Heuser and Reese, 1973; Neher, 2010). This constant membrane trafficking during the essential step of membrane endocytosis - which necessarily precedes formation of new vesicles to be re-filled with $\mathrm{ACh}$ - requires adequate space along the terminal branch to enable such continuous fusion and re-uptake of membranous material. Accordingly, a smaller number of vesicles relative to the same length of nerve terminal branching must be viewed as a distinct advantage in highly active neuromuscular systems as it would more favorably accommodate the rapid recycling of the pool of readily releasable pre-synaptic vesicles. This need for adequate space to enable large volume, rapid vesicular recycling during periods of high neuromuscular activity may also account for the more dispersed distribution of ACh-containing vesicles within the nerve terminal arborization of trained animals. Consistent with our other significant training-related adaptations, this reduced amount of vesicular staining relative to branch length was noted only among younger animals. It is interesting to note that in the data reported here, endurance training consistently - at least among young animals - remodeled pre-synaptic variables including both vesicles and the branches to which they are tethered. Previously it was reported that resistance training, i.e. weight lifting, resulted in adaptations only among the post-synaptic component of the NMJ and as evident here with endurance training, synaptic alterations occurred without accompanying changes in the size of myofibers (Deschenes et al., 2015).

Recall that the principal research question to be addressed in the present investigation was to determine if pre- to post-synaptic coupling could be maintained in the face of the relatively abrupt NMJ remodeling that is triggered by exercise training and whether aging would alter such adaptability. The data generated here indicate that despite significant training-induced alterations in both pre- and post-synaptic morphology, coupling remains stable, and that aging does not moderate the durability of this synaptic architecture. These facts were found to be true whether this coupling was assessed as the area occupied by immunofluorescently stained ACh receptors relative to total nerve terminal branch length, or as the relative overlap of stained pre-synaptic vesicles, i.e., release sites, with post-synaptic receptors or binding sites. Such effective coupling of pre- and post-synaptic components of the NMJ is likely due to a combination of the physical connection of pre- and postsynaptic membranes by tough but thin synapsespanning proteins such as laminin (Nishimune et al., 2004, 2008; Samuel et al., 2012), and transmembrane communication made possible by the exchange of nontransmitter molecules including neurotrophic factors and such as, among others, glial-derived neurotrophic factor, brain-derived neurotrophic factor, as well as agrin (Gyorkos and Spitsbergen, 2014; Schinder and Poo, 2000; Wu et al., 2010).

Clearly indicated in the data gathered in this study is that the influence of aging on NMJ structure does not necessarily follow the same time course as the effects of aging on the myofibers on which those NMJs reside. 
More specifically, no main effect for aging was identified for even a single parameter of NMJ morphology; this was true for NMJs assessed in both the plantaris and the EDL. This is in stark contrast with our statistical findings of myofiber profiles for those same muscles. In fact, in the plantaris five of the variables examined exhibited a main effect of aging confirming that aged rats showed signs of sarcopenia including myofiber atrophy and fiber-type conversion which typically results in slower contractile velocity and diminished power. This is best reflected in aging-related decline in the percentage of Type IIB fibers, as well as the relative area of the whole muscle occupied by those same Type IIB fibers.

Assessment of the similarly fast-twitch EDL muscle revealed only two main effects for aging and they both related to atrophy. That is, with fiber cross-sectional area collapsed across fiber types, aged myofibers were smaller than young adult ones; this was also found to be the case specifically for Type IIX myofibers. The same number of variables displaying a main effect for endurance training was noted in the EDL as found in the plantaris, although they were not the same ones. It is noteworthy that myofiber profiles of the EDL were affected both by aging and exercise training in view of the fact that no significant influence of either of those factors were noted among NMJs quantified in the same EDL muscles. These findings strongly suggest that modifications of the neuromuscular system are not necessarily accompanied by changes in both the NMJs and the myofibers on which those synapses reside.

All told, the findings presented here indicate that muscle function during treadmill running, i.e., duty cycle, likely plays the most prominent role in governing traininginduced NMJ remodeling. This is borne out by the fact that NMJs of the plantaris exhibited considerable morphological adaptation while those of the EDL, despite a very similar fiber-type composition as the plantaris, did not. Moreover, it was confirmed in this investigation that aging does, indeed, influence the neuromuscular system's capacity for training-induced plasticity as only young adult NMJs demonstrated significant reconfiguration as a result of treadmill running. Also presented here is evidence that age- and exerciseinduced adaptations of the two components of the neuromuscular system - myofibers and the NMJs that activate those fibers - do not necessarily coincide. But perhaps the most intriguing finding of this project - and the one that served as the main focus - is that despite the considerable overall remodeling of the NMJ, pre- to post-synaptic coupling assessed both as endplate area relative to nerve terminal branch length, and as the degree of overlap between stained pre-synaptic AChcontaining vesicles and stained post-synaptic receptors that bind that neurotransmitter, remains intact. This speaks to the fundamental importance of maintaining proper synaptic communication in order to assure effective neuromuscular function.

Acknowledgments-This research was supported by funding from NIH grant R15 060637-04, and The Foundation for Aging Studies and Exercise Science Research.

\section{REFERENCES}

Anderton BH, Breinburg D, Downes MJ, Green PJ, Tomlinson BE, Ulrich J, Wood JN, Kahn J (1982) Monoclonal antibodies show that neurofibrillary tangles and neurofilaments share antigenic determinants. Nature 298:84-86.

Andonian MH, Fahim MA (1988) Endurance exercise alters the morphology of fast- and slow-twitch rat neuromuscular junctions. Int J Sports Med 9:218-223.

Arias E (2006) United States life tables, 2003. National vital statistics reports. Hyattsville, MD: National Center for Health Statistics.

Cheng A, Morsch M, Murata Y, Ghazanfari N, Reddel SW, Phillips WD (2013) Sequence of age-associated changes to the mouse neuromuscular junction and the protective effects of voluntary exercise. PLoS One 8:e67970.

Delp MD, Duan C (1996) Composition and size of type I, IIA, IID/X, and IIB fibers and citrate synthase activity of rat muscle. J Appl Physiol (1985) 80:261-270.

Deschenes MR, Maresh CM, Crivello JF, Armstrong LE, Kraemer WJ, Covault J (1993) The effects of exercise training of different intensities on neuromuscular junction morphology. J Neurocytol 22:603-615.

Deschenes MR, Judelson DA, Kraemer WJ, Meskaitis VJ, Volek JS, Nindl BC, Harman FS, Deaver DR (2000) Effects of resistance training on neuromuscular junction morphology. Muscle Nerve 23:1576-1581.

Deschenes MR, Roby MA, Glass EK (2011) Aging influences adaptations of the neuromuscular junction to endurance training. Neuroscience 190:56-66.

Deschenes MR, Hurst TE, Ramser AE, Sherman EG (2013) Presynaptic to postsynaptic relationships of the neuromuscular junction are held constant across age and muscle fiber type. Dev Neurobiol 73:744-753.

Deschenes MR, Sherman EG, Roby MA, Glass EK, Harris MB (2015) Effect of resistance training on neuromuscular junctions of young and aged muscles featuring different recruitment patterns. J Neurosci Res 93:504-513.

Fahim MA (1997) Endurance exercise modulates neuromuscular junction of C57BL/6NNia aging mice. J Appl Physiol (1985) 83:59-66.

Glenn GM, Laughlin MH, Armstrong RB (1987) Muscle blood flow and fiber activity in partially curarized rats during exercise. J Appl Physiol (1985) 63:1450-1456.

Gonzalez-Freire M, de Cabo R, Studenski SA, Ferrucci L (2014) The neuromuscular junction: aging at the crossroad between nerves and muscle. Front Aging Neurosci 6:208.

Gyorkos AM, Spitsbergen JM (2014) GDNF content and NMJ morphology are altered in recruited muscles following highspeed and resistance wheel training. Physiol Rep 2:e00235.

Heuser JE, Reese TS (1973) Evidence for recycling of synaptic vesicle membrane during transmitter release at the frog neuromuscular junction. J Cell Biol 57:315-344.

Jang YC, Van Remmen H (2011) Age-associated alterations of the neuromuscular junction. Exp Gerontol 46:193-198.

Kononenko NL, Haucke V (2015) Molecular mechanisms of presynaptic membrane retrieval and synaptic vesicle reformation. Neuron 85:484-496.

Kwon SE, Chapman ER (2011) Synaptophysin regulates the kinetics of synaptic vesicle endocytosis in central neurons. Neuron 70:847-854.

Laughlin MH, Korthuis RJ, Sexton WL, Armstrong RB (1988) Regional muscle blood flow capacity and exercise hyperemia in high-intensity trained rats. J Appl Physiol (1985) 64:2420-2427.

Neher E (2010) What is rate-limiting during sustained synaptic activity: vesicle supply or the availability of release sites. Front Synaptic Neurosci 2:144.

Nishimune H, Sanes JR, Carlson SS (2004) A synaptic laminincalcium channel interaction organizes active zones in motor nerve terminals. Nature 432:580-587.

Nishimune H, Valdez G, Jarad G, Moulson CL, Muller U, Miner JH, Sanes JR (2008) Laminins promote postsynaptic maturation by 
an autocrine mechanism at the neuromuscular junction. J Cell Biol 182:1201-1215.

Pearson J, Sabarra A (1974) A method for obtaining longitudinal cryostat sections of living muscle without contraction artifacts. Stain Technol 49:143-146.

Prakash YS, Miller SM, Huang M, Sieck GC (1996) Morphology of diaphragm neuromuscular junctions on different fibre types. J Neurocytol 25:88-100.

Rizzoli SO (2014) Synaptic vesicle recycling: steps and principles. EMBO J 33:788-822.

Robbins N (1992) Compensatory plasticity of aging at the neuromuscular junction. Exp Gerontol 27:75-81.

Rosenheimer JL (1990) Ultraterminal sprouting in innervated and partially denervated adult and aged rat muscle. Neuroscience 38:763-770.

Rudolf R, Khan MM, Labeit S, Deschenes MR (2014) Degeneration of neuromuscular junction in age and dystrophy. Front Aging Neurosci 6:99.

Samuel MA, Valdez G, Tapia JC, Lichtman JW, Sanes JR (2012) Agrin and synaptic laminin are required to maintain adult neuromuscular junctions. PLoS One 7:e46663.
Schinder AF, Poo M (2000) The neurotrophin hypothesis for synaptic plasticity. Trends Neurosci 23:639-645.

Seidi AH (2014) Regulation of conduction time along axons. Neuroscience 276:126-134.

Tomas J, Fenoll R, Mayayo E, Santafe M (1990) Branching pattern of motor nerve endings in a skeletal muscle of the adult rat. J Anat 168:123-135.

Turtorro A, Witt WW, Lewis S, Hass BS, Lipman RD, Hart RW (1999) Growth curves and survival characteristics of the animals used in the Biomarkers of Aging Program. J Gerontol A Biol Sci Med Sci 54:B492-B501.

Valdez G, Tapia JC, Kang H, Clemenson Jr GD, Gage FH, Lichtman JW, Sanes JR (2010) Attenuation of age-related changes in mouse neuromuscular synapses by caloric restriction and exercise. Proc Natl Acad Sci U S A 107:14863-14868.

Waerhaug O, Dahl HA, Kardel K (1992) Different effects of physical training on the morphology of motor nerve terminals in the rat extensor digitorum longus and soleus muscles. Anat Embryol (Berl) 186:125-128.

Wu H, Xiong WC, Mei L (2010) To build a synapse: signaling pathways in neuromuscular junction assembly. Development 137:1017-1033.

(Accepted 2 December 2015)

(Available online 19 December 2015) 